\title{
An Effective Reliability Evaluation Method for Power Communication Network Based on Community Structure
}

\author{
Qi Li, Zehong Cao *, Member, IEEE, M. Tanveer, Senior Member, IEEE, H.M. Pandey, Senior Member, IEEE, \\ and Chen Wang
}

\begin{abstract}
The reliability evaluation of power communication network is beneficial for the improvement of stable operation of the power system and the robustness of the power grid. However, the existing reliability evaluation models of the power communication network cannot meet the current situation of timeliness performance, due to rapidly increasing scale and complexity of information across varying services. In this study, we used the complex network theory to analyze the structure of power communication network and constructed the evaluation index of node (link) reliability of power communication network based on community reliability. Compared with the traditional reliability indexes, our index not only considers the influence of the environment of the node (link) on the single structure of the power communication network, but also possess the reliability evaluation rate of the node (link), which have the opportunities for improving the performance of the reliability evaluation of the wide-area power communication network. To verify the rationality of the index, we developed random, low reliability and high-betweenness deliberate attacks to attack the designated node (link), and compared the network efficiency before and after the attack. Based on the simulation results, it can verify the rationality and superiority of our proposed evaluation index.
\end{abstract}

Index Terms-complex network, security, community structure, power communication network, reliability.

\section{INTRODUCTION}

Power system is a typical system of information and energy fusion. Each part of the power system needs the support of the power communication network to ensure the conversion of electricity and other energy. The basic mode of power system includes dispatch, participation, power market, power consumption, power distribution, power transmission, power generation and so on. It can transform different forms of energy into electrical energy and transport to a place where electricity is needed. In the process of power transmission, the power communication network carries the services of communication control, basic information transmission and

Qi Li is with Department of Computer Science and Engineering, Shaoxing University, Zhejiang, China.

Zehong Cao is with the Department of Discipline of ICT, School of Technology, Environments and Design, University of Tasmania, Hobart, Australia. * Corresponding author (e-mail: Zehong.Cao@utas.edu.au or zhcaonctu@gmail.com)

M. Tanveer is with Discipline of Mathematics, Indian Institute of Technology Indore, Indore, India.

H.M. Pandey is with Department of Computer Science, Edge Hill University, Ormskirk, United Kingdom.

Chen Wang is with College of Computer Science, Chongqing University, Chongqing, China. effective state perception [1], [2]. With the construction of the wide area smart grid, more power system services need to be transmitted through the power communication network. The dependence of power system on communication network is increasing, and the influence of communication network failure on power system is becoming more and more serious, therefore, the research work for the reliability evaluation of power communication network is paid more and more attention.

The reliability of power communication network nodes (links) as an effective measure of the reliability of communication networks reflects the ability of different network units to maintain communication in various environments, identify weaknesses in the network, and provides data support for network planning and risk management. The existing power communication network node (link) reliability indicators include connectivity [3], integrity [4], viscosity [5], discrete number [6], natural connectivity [7] and so on. With the expansion of the scale of the power communication network, these indicators cannot provide real-time evaluation of the node (link) reliability of the wide area power communication network due to the greatly increased computational time complexity.

The concept of network reliability was first proposed by Lee [8]. Most of the early researchers preferred Lee's evaluation model based on graph theory and physical failure of equipment, focusing on how to improve various algorithms to reduce complexity. Since the 1980s [9], network capacity and congestion began to become the focus of attention. Since the 1990s, network reliability research has become a hot topic, more and more reliability-related concepts have been put forward, such as performance reliability, availability, completion [10].

At present, China's power grid is in a period of high-speed development, especially in recent years, the power grid is vigorously developing ultra-high voltage (UHV) power grid [11]. The UHV power grid line has a long distance and a wide range of control. The rapid development of the power grid has brought great challenges to the support ability of the power communication network. With the continuous construction and development of power communication network, the network scale is increasing, the number of power communication core business will also be increasing, the power communication network system is becoming more complex. The traditional power communication network reliability assessment method cannot 
meet the demand of the current power communication network in terms of algorithm complexity and time complexity, and it is difficult to analyze the reliability of power communication network in time and effectively. In this paper, we combine the topological characteristics of power communication network and the theory of complex network, establish efficient node (link) reliability index of power communication network, and improve the calculation method of all-terminal reliability of power communication network, make it suitable for the reliability evaluation of wide area power communication network. Our main contributions can be summarized as follows:

- We analyzed the network structure and topological characteristics of the power communication network and provided proof that the power communication network possesses a typical community structure. Based on these measurements, a fast unfolding algorithm is designed, to mine the power communication network quickly and identify the community structure.

- Based on the community model, we proposed a reliability node (link) evaluation method of power communication network, by combining the community reliability characteristics. With argument the reliability index of communication node and link, it can effectively find the important communication nodes (links) in the network, to improve the reliability of these nodes (links) and achieve the stable operation of the power grid.

- In the experiment, random attack, low-reliability attack, and high-betweenness attack are used to attack the designated nodes and links of the power communication network. Comparing the network efficiency before and after the attack, the simulation results can verify the rationality of the reliability evaluation index in our study.

The rest of this paper is organized as follows; Section II, briefly outlines a list of related works and our motivation. Section III, modeling and analysis of power communication network topology. Detailed reliability method of node (link) in Power Communication Network is presented in Section IV. Section V, presents the experiment results, and comparison with completing algorithms. Finally, this paper is concluded in Section VI.

\section{RELATED WORK}

Power communication network is the communication network of power system [12], which carries all the business of power production information and management. With the continuous improvement of the automation level of power system [13], the power communication network plays an increasingly important role in power production and dispatch. The reliable operation of power communication is related to the normal production of power system. Therefore, it plays an important role in the reliability of power communication network.

\section{A. Community detection}

The community structure of the network is one of the common features that researchers have found in many actual networks in recent years [14]. In short, the nodes of the network are divided into different groups, where the intergroup nodes are closely connected to the intergroup nodes and the intergroup node connections are sparse compared to the intergroup connections. The study of the topological structure of the community structure in the network is helpful to understand the characteristic structure and propagation characteristics of the whole complex network.

The research on the network community mainly focuses on the community mining algorithms. The network can be divided into non-overlapping communities and overlapping communities [15], so the corresponding mining algorithm is not the same. For non-overlapping communities, the author put forward the community mining algorithm based on the modularityFastGN [16]. On the basis of FastCN algorithm, Clauset et.al [17] uses heap data structure to calculate and update the modularity of the network. A new greedy algorithm is proposed to reduce the time complexity of community mining to a linear time. Liu et.al makes the community mining algorithm more suitable for specific business scenarios by improving the similarity index [18]. For overlapping communities, Li et.al [19] proposes a clique percolation method, which obtains overlapping communities by analyzing the overlapping matrix of factions. Steve Gregory put forward a lot of algorithms about overlapping community detection, including CONGA algorithm [20], which takes into account the local betweeness characteristics between nodes, the algorithm can quickly improve the community overlap detection. The emergence of these algorithms has greatly enriched the community mining of modern complex networks, different business scenarios need to choose different algorithms to implement. The idea of constructing node (link) reliability index is shown in Fig. 1 .

\section{B. Reliability of Power Communication Network}

Modern public communication network [21] is a communication system consisting of communication equipment and protocol. It consists of three parts: transmission, exchange and terminal. Since the establishment of the first recognized public communication network in the late 1960s, the communication network has made great progress. In order to ensure that the public communication network can reliably transmit information, the research on the reliability of the public communication network has become a hot topic for scholars. The communication public network is an integrated system composed of software and hardware, the reliability of the public network mainly includes hardware reliability [22] and software reliability [23]. Scholars mainly measure the reliability of network topology structure from the four indicators of network invulnerability, survivability, availability and completeness. For the study of software reliability, the researchers have defined software reliability indicators such as software reliability function, software failure distribution function, software failure density function.

Compared with the research results of public communication network reliability, the research on power communication network reliability has accumulated less, and Most research institutes refer to the results of public network reliability 
research [24], [25]. Because of the characteristics of long communication distance, fine business granularity, strong realtime and high security reliability, the power communication provides "best effort" service relative to the public network. As a special power communication network in power system, power communication network requires high reliability services to ensure that power communication services can access power communication network at any time. At the same time, in order to meet the real-time characteristics of power communication, the timeliness of power communication network reliability evaluation method is also highly required. The reliability of power communication network is mainly developed from two aspects: network structure reliability [26] and service reliability [27]. Traditional reliability research of power communication network is mainly based on graph theory and probability theory. The author [28], [29] regards communication network as a weighted undirected graph. Based on graph theory, the reliability of power communication network topology is studied and optimized. Because of the development of computer technology, Monte Carlo simulation technology [30] and genetic algorithm [31] are also widely used in the reliability research of power communication network. Yang et.al [31] explores the reliability of power communication network using improved genetic algorithm.

With the deepening of research and the development of power communication network theory, scholars began to consider combining power communication network service performance indicators to evaluate the reliability of power communication network. The paper comprehensively considered the impact of service risk indicators on the reliability of power communication network, and improved the reliability of power communication network by means of service rerouting. Many research results are limited to small-scale power communication networks, and lack of efficiency and accuracy for reliability evaluation of wide-area power communication networks.

In summary, scholars at home and abroad have made remarkable achievements in the field of reliability evaluation of power communication network and complex network community structure. However, due to the real-time requirement of power business and the development of wide area power communication network, the traditional reliability evaluation method of power communication network has been unable to meet the timeliness requirement of reliability evaluation. Combining the community structure of complex network with the reliability evaluation method of power communication network, it is of practical significance to study the reliability evaluation method of power communication network based on community structure.

\section{CONSTRUCTION OF THE COMMUNITY STRUCTURE OF POWER COMMUNICATION NETWORK}

In order to facilitate the operation, control and management of regional power grid. Generally, the power communication network is managed by the way of zoning management. Zoning management makes some faults or control behaviors in the partition have little influence on other partitions. Therefore, the

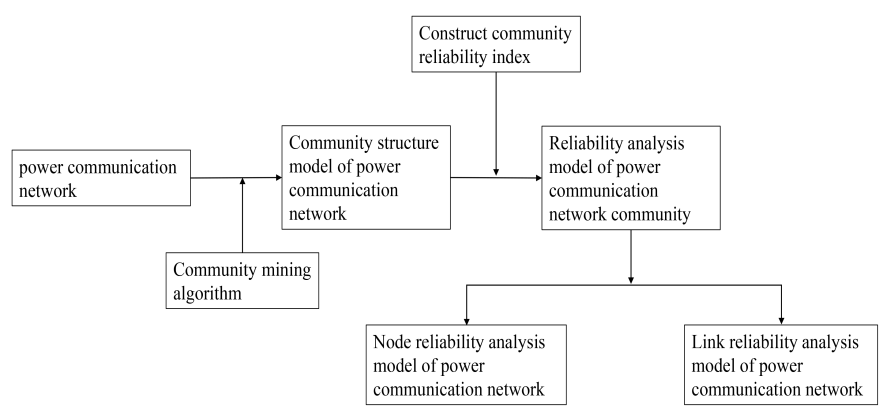

Fig. 1. The idea of constructing node (link) reliability index

reasonable partition is the premise of scientific and effective operation and control of power grid. In China, the division of power communication network is usually based on the division of administrative region. Because of the large consumption of electric power in administrative region, a lot of control information is needed to ensure the normal operation of urban power supply and distribution. It is necessary to build a complex power communication network. Therefore, the connection tightness of the network in the administrative region is usually higher than that between the administrative regions. This feature is quite similar to the community structure in complex networks, and the way of community division can better find the optimal partition from the network topology itself. Therefore, it is of great significance and value to study the partition of power communication network by community mining [32], [33].

Community mining algorithms are mainly divided into two categories: agglomeration [34], [35] and decomposition [36]. The basic idea of agglomeration algorithm is to add links for nodes with higher similarity. In contrast, the decomposition algorithm usually removes links between nodes with lower similarity. The community division needs to define the modularity [37], the modularity is the method of measuring the strength of the structure of the network community. The higher the value, the better the effect of community division, on the contrary, the community division effect is poor and the lower the communication robustness of the nodes in each community.

$$
\begin{aligned}
& Q=\frac{1}{2 M} \sum_{i j}\left(P_{i j}-\frac{t_{i} t_{j}}{2 M}\right) \delta\left(C_{i}, C_{j}\right) \\
& \delta\left(C_{i}, C_{j}\right)= \begin{cases}1 & C_{i}=C_{j} \\
0 & C_{i} \neq C_{j}\end{cases} \\
& M=\frac{1}{2} \sum_{i, j} P_{i j} \\
& t_{i}=\sum_{j} P_{i j}
\end{aligned}
$$

For modularity calculation, as shown in Formula (1). $P_{i j}=$ 1 denotes that there is a link between node $v_{i}$ and node $v_{j}$, otherwise it is $0 . M$ represents the sum of all optical fibers in the network, and $t_{i}$ represents the sum of all optical fibers connected to node $i$. Formula (1) represents the proportion of 


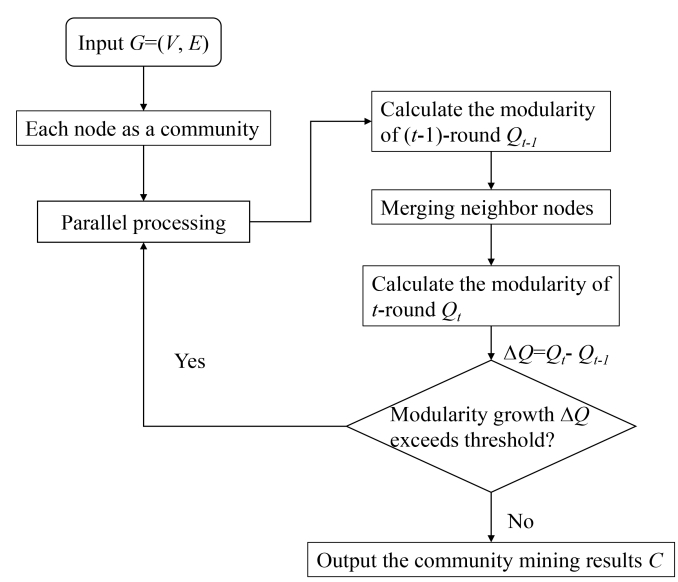

Fig. 2. The flow diagram of algorithm

links connecting the internal nodes the community in the network, minus the expected value of the proportion of arbitrarily connecting the two nodes under the same community.

The Fast Unfolding algorithm [38] can be used to cluster closely connected communication stations to form a communication community. The algorithm is a heuristic algorithm that has the advantage of high efficiency and good division effect. It is a typical unsupervised learning algorithm. the stability of the result is higher than the traditional data mining algorithm. The traditional Fast Unfolding algorithm is an iterative algorithm, the principle of which is to continuously reconstruct the community and the $Q$ value of the whole network after division is constantly increased. The algorithm consists of two stages. The first stage is called modularity optimization, which divides each node into the community where it is adjacent to the node, so that the $Q$ value is constantly increased; The second stage, called community aggregation, is primarily the aggregation of the first step of the community into a node, i.e. the network is reconstructed according to the community structure generated in the previous step. Repeat the above process until the structure of the network does not change [38], the main steps of the algorithm are as follows.

Step 1. Assuming that there are $N$ nodes in the network. In the initial state, a community number $i$ is assigned to each node $i$, then there are $N$ communities in the network.

Step 2. For each node $i$, try to add its adjacent node $j$ to the corresponding community of $i$. If the modularity change $\triangle Q$ of the network exceeds the threshold value after joining, then accept the change. On the contrary, $j$ still belongs to the original community.

Step 3. When the number of iterations is less than the total number of iterations, repeat Step 2.

The Fast Unfolding algorithm is improved to a parallelization program because the association and separation between the nodes are independent. It can be applied to large-scale community mining algorithms, and the time complexity is also greatly improved. Parallelization of the fast unfolding algorithm improves the efficiency of the algorithm. The flow chart of parallel fast unfolding algorithm in Fig. 2, and the corresponding pseudo code is shown in algorithm 1 .

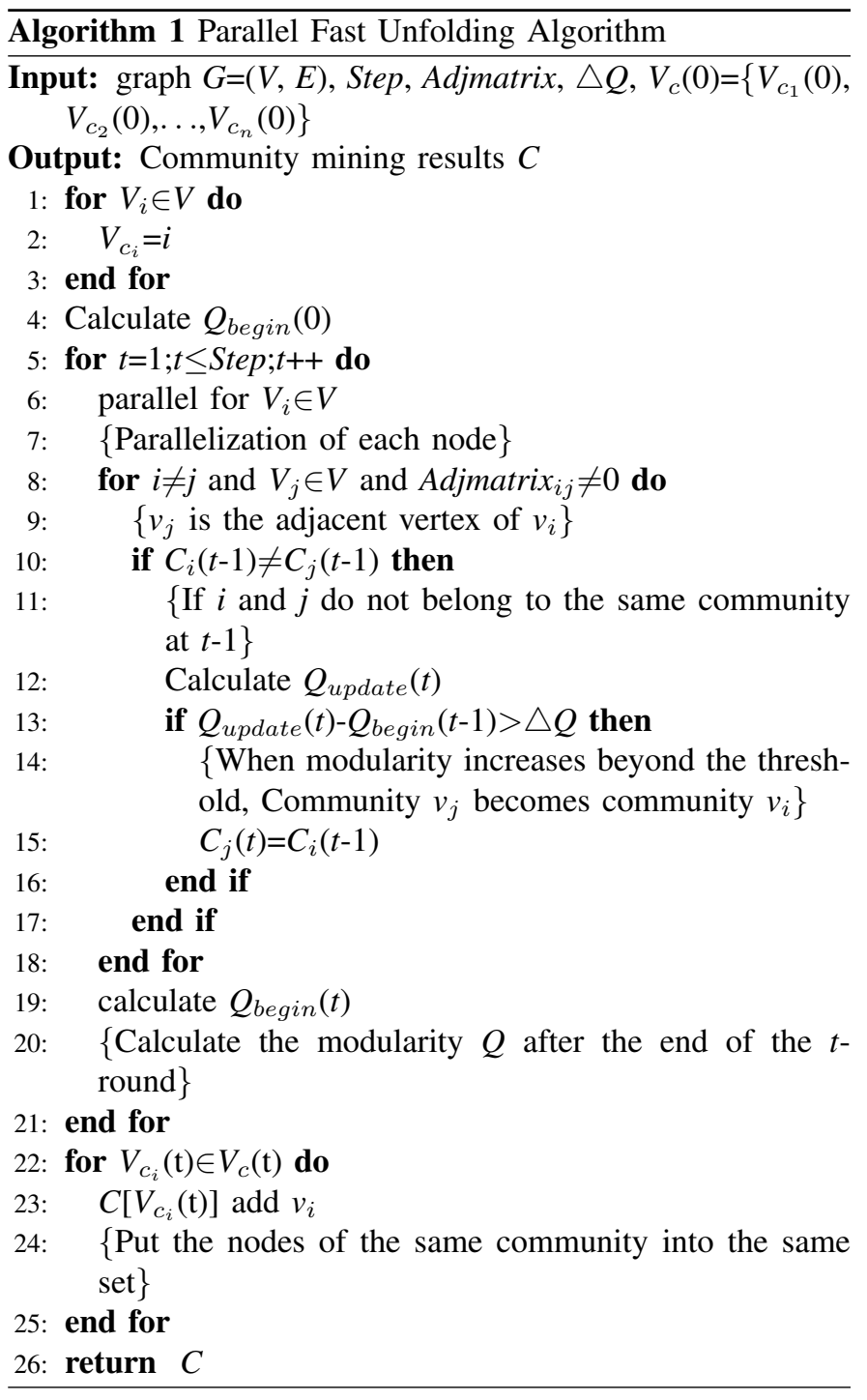

The basic principle of the algorithm is that the community ownership of a node in the $t$ round is determined by the community of the neighbor node of the $t-1$ round in the Step round iterative process. In this way, the community division state of $t-1$ round can be shared in distributed environment. Calculating the community which each node belongs is an independent events that can be parallelized in the $t$ round. In the iteration process of establishing power communication network community model based on parallel fast unfolding algorithm, the number of communities decreases gradually with the merging of nodes, the modularity of the whole network increases gradually, and finally tends to ease.

We divide the structure of power communication network in 3 threads through the traditional parallelized Fast Unfolding algorithm (best algorithm) and the improved parallelized Fast Unfolding algorithm (parallelized Fast Unfolding algorithm), and compare the convergence of the number and module of the associations in the network under different iteration times, to prove the availability and efficiency of the algorithm.

In the process of establishing the model of the power communication network community, the relationship between the number of communities and the number of iterations is 


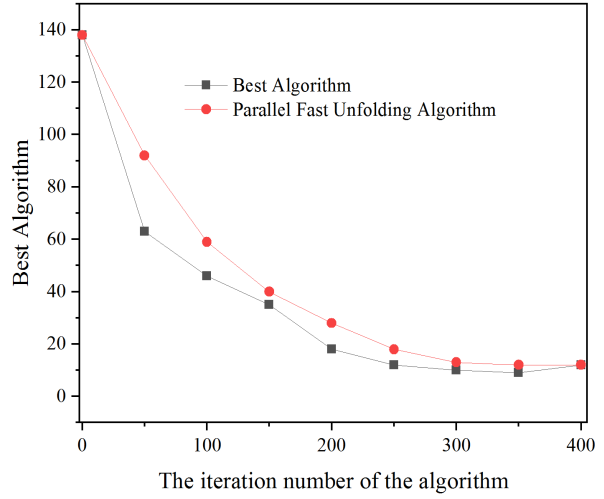

Fig. 3. Changes in the number of communities in the process of community mining by different algorithms

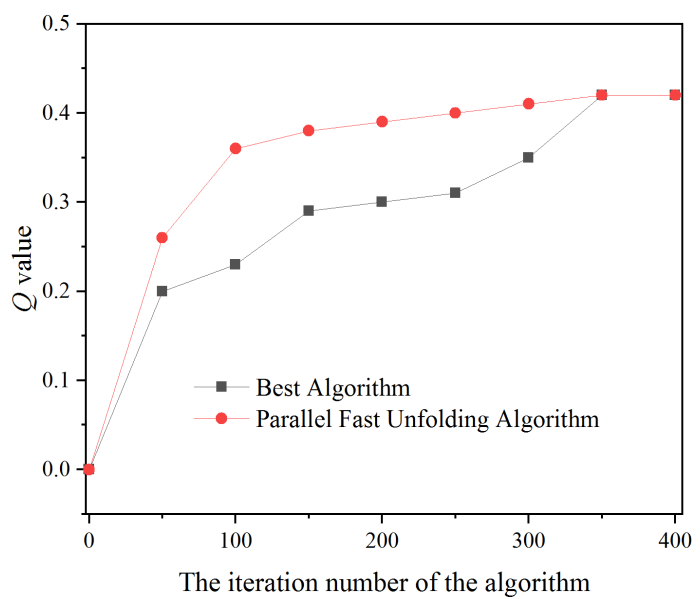

Fig. 4. Modularity Value of different algorithms in the Process of community mining

shown in Fig. 3, the initial number of communities is 138, and the improved parallelized Fast Unfolding algorithm has passed 350 iterations and finally converges to 10 communities. In the convergence trend of the algorithm, the number of communities converges quickly in the early stage and gradually stabilizes in the later stage. The improved parallelized Fast Unfolding algorithm has the characteristics of fast convergence speed, high efficiency. The improvement of community mining efficiency will effectively improve the efficiency of the reliability evaluation of power communication network in the follow-up process, and make it possible to evaluate the real-time reliability of the wide area power communication network.

Modularity is one of the important bases to measure whether the division of community structure is reasonable. In the process of mining the power communication network communities, the change of the modularity of the power communication network is shown in Fig. 4. with the decrease of the number of communities, the modularity value continues to grow larger, reflecting that each step of the community consolidation is the preferred choice in the process of iteration. The consolidation of associations plays an obvious role in the promotion of the modularity value. After the community convergence is the final number, the modularity value is also converged to 0.421 ,

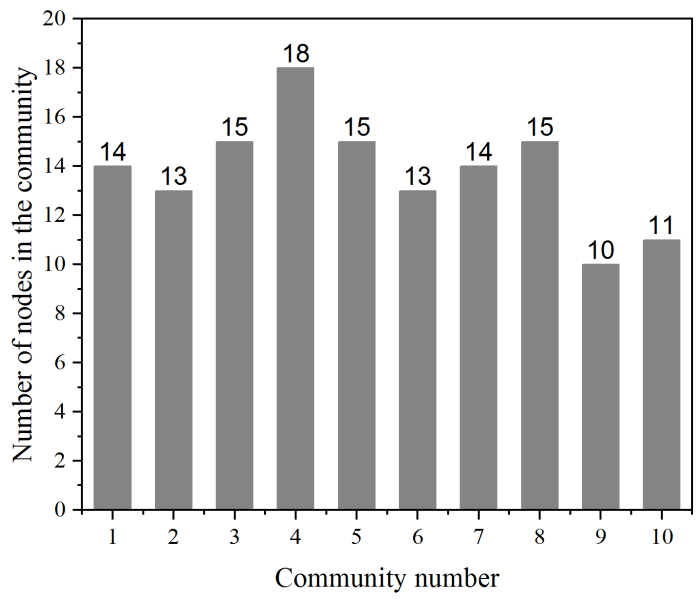

Fig. 5. Distribution of nodes of communities

and the improved parallelized Fast Unfolding algorithm finally converges the modularity value after 350 iterations.

After the community division, the number of nodes in each community of power communication network is shown in Fig. 5. The number of nodes in the community is between $[10,18]$. The distribution of nodes in the community is relatively uniform, there is no isolated community. Therefor, the community division method has good effect. Such uniform distribution is very helpful for the parallel analysis of subsequent reliability evaluation. If there is a problem that the maximum number of nodes in the community is far greater than the minimum number of nodes in the community, which lead to the data skew problem in the process of parallel processing. The problem of data skew can seriously affect the parallelism of the program, the reliability evaluation of the largest community determines the operation efficiency of the whole program. Finally, the whole power communication network is cut into 10 partitions by the community mining model. These partitions are closely connected internally and loosely connected between them. The variance of the number of nodes in each partition is small, which is convenient to analyze and mine the properties of each partition.

\section{Proposed Node (link) Reliability Method}

\section{A. Reliability Analysis of Power Communication Network}

The reliability of nodes and links in different communities should be measured differently due to the different communities. In order to reflect this difference, we first define the reliability of community structure. For the community reliability, we use the vulnerable set of each community to measure the reliability of different communities. The meaning of the vulnerable set of a community is that when all edges of the vulnerable set are interrupted due to random fault or deliberate attack, the community will be disconnected from all communities in the network, forming an independent connected branch. The more the number edges in vulnerable set is, the less likely it is that the community will be disconnected from other communities, and the more reliability it will be, and vice versa. 


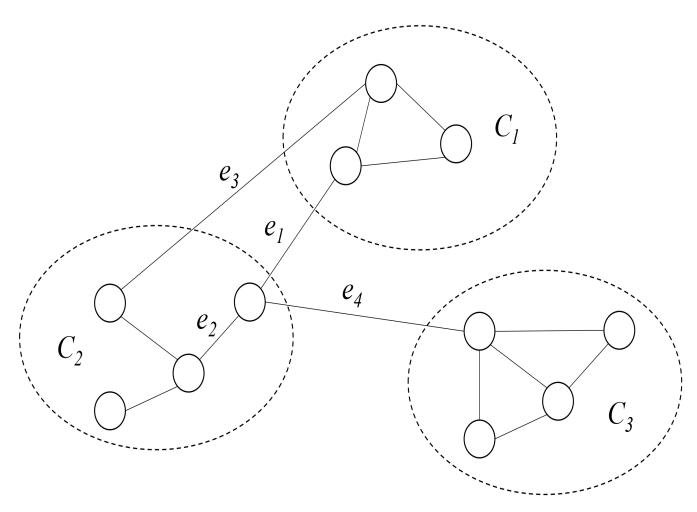

Fig. 6. An example of community vulnerable set

Given a power communication network $G=(V, E)$, its community structure has been determined. Where $V$ is the vertex set and $E$ is the edge set. $C_{x}(x \in G)$ denotes the community set and there is no intersection between communities. The vulnerable set of community $x$ and $y$ is defined as: $V_{c_{x y}}=\left\{(u, v) \in E, u \in C_{x}, v \in C_{y}, x \neq y\right\}$, As shown in fig. 6 . $V_{c_{12}}=e_{1}, e_{2}$. If all edges of $V_{c_{x y}}$ are destroyed, the community $x$ loses direct contact with the community $y$. Therefore, the topological reliability index $v(x, y)$ of community $x$ and $y$ is defined as.

$$
v(x, y)=\left|V c_{x y}\right|
$$

Where $\left|V_{c_{x y}}\right|$ denotes the number of elements in $V_{c_{x y}}$. For community $x$, its vulnerable set $V_{c_{x y}}$ can be defined as follows.

$$
V_{c_{x}}=\bigcup_{y \in G} V_{c_{x y}}
$$

Therefore, the topological reliability $C R\left(c_{i}\right)$ of community $i$ can be defined as.

$$
C R\left(c_{i}\right)=\frac{\left|V_{c_{i}}\right|}{\sum_{x \in G}\left|V_{c_{x}}\right|}
$$

When community $C_{i}$ is closely related to external communities, there are many elements in vulnerable set $C_{i}$, and the disconnection of part of edges can not make community $C_{i}$ become a separate connected branch or island. Therefore, its reliability is higher. As shown in Fig.4, The removal of any link $e_{1}$ or $e_{2}$ do not make the community $C_{1}$ or $C_{2}$ become an island, but the removal of $e_{4}$ can make the community $C_{3}$ become an island. It can be concluded that the reliability of community $C_{1}$ and $C_{2}$ is higher than that of $C_{3}$.

Community reliability is defined to show that nodes (links) in different communities have different reliability due to the influence of the surrounding environment. For nodes (links) in low-reliability communities, due to the faults of some fragile (nodes) links, the ability to communicate with nodes in the overall network will be lost. By constructing community reliability, the reliability of the environment in which different nodes (links) are located can be measured.

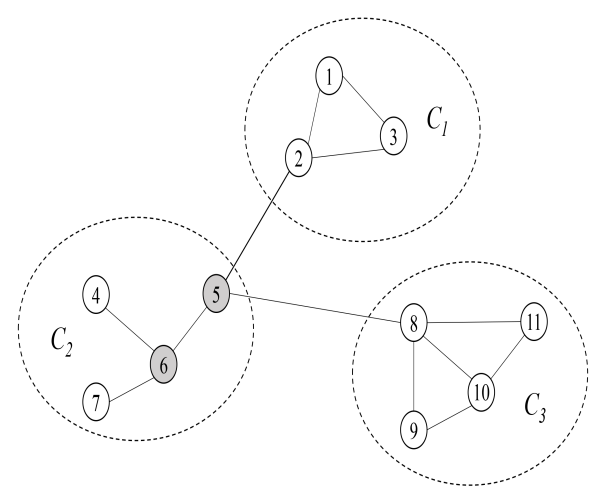

Fig. 7. Node comparison in community structure

\section{B. Node Reliability Analysis of Power Communication Net- work}

Different businesses have different requirements for realtime and reliability of power communication network. Power communication network is mainly divided into trunk transmission network and metropolitan area network. Trans-regional business dispatching and management is mainly carried by trunk transmission network, and Power dispatching and information transmission in the region are mainly transmitted by metropolitan area network. In these two kinds of power communication network, there are communication nodes such as power plant, power distribution station, substation and power dispatch communication center. These nodes can be divided into core nodes, backbone nodes and access nodes according to different importance. Once the node is paralyzed, it will cause huge loss to the network service transmission. Therefore, it is necessary to analyze the reliability of the communication node in the power communication network.

Community structure has an important impact on the reliability evaluation of nodes. As shown in Fig. 7, node 5 and node 6 have the same degree and should have the same reliability. However, after the community is divided, the attributes of these two nodes have changed a lot. Node 5 becomes the boundary node, which is the bridge node between different communities. Node 6 becomes the internal node of the community. The boundary nodes of communities not only undertake the information transmission within communities, but also undertake the information transmission between communities. They are in a key position in the whole network, Therefore, when measuring the reliability of different nodes, the reliability of the community in which the node is located should be considered, and the key position of the node in the whole network should also be considered.

Based on the above analysis, we put forward an evaluation index of node reliability based on community structure (CNR), which combines the reliability of the community where the node is located and the reliability of edge position where the node is located. The definition is shown in the following formula.

$$
C N R\left(v_{i}\right)=\sum_{k=1}^{|C|} \delta(i, k) C R\left(C_{k}\right) \times B R\left(v_{i}\right)
$$


Where

$$
\begin{gathered}
B R\left(v_{i}\right)=\left\{\begin{array}{c}
\frac{1}{\sum_{t}^{t \in \lambda_{i}^{i n}} e_{i t}}\left|\mu_{i}^{a d j}\right|=0 \\
\frac{\prod_{k \in \mu_{i}^{a d j}} C R\left(C_{k}\right)}{\sum_{t}^{t \in \lambda_{i}^{i n}} e_{i t}}\left|\mu_{i}^{a d j}\right| \neq 0
\end{array}\right. \\
\delta(i, k)=\left\{\begin{array}{c}
1 i \in k \\
0 \text { otherwise }
\end{array}\right.
\end{gathered}
$$

Where $\sum_{k=1}^{|C|} \delta(i, k)$ is used to measure the reliability of the community where the node is located. And $-C$ - represents the number of communities in the network. In order to measure the reliability of edge position where the node is located. We define the reliability of edge position where the node is located $B R\left(v_{i}\right) . \mu_{i}^{a d j}$ denotes the community set directly connected to node $i .\left|\mu_{i}^{a d j}\right|$ denotes the number of elements in $\mu_{i}^{a d j}$. $\lambda_{i}^{i n}$ denotes the neighbor nodes in the community which the node $i$ belongs. When there is an edge between node $i$ and $j$, $e_{i j}=1$, otherwise, $e_{i j}=0 . B R\left(v_{i}\right)$ means that if a node $i$ as a bridge between different communities, then the reliability of its community edge is related to the reliability of the communities it connects. As shown in Fig. 5, the edge-position reliability of node 5 is related to community $C_{1}$ and $C_{3}$. Since nodes 2 and 8 belong to different communities with different degree of tightness, the edge-position reliability should be different. In order to show the difference, $\sum_{t}^{t \in \lambda_{i}^{i n}} e_{i t}$ is introduced in Formula 6 to indicate the degree of tightness between boundary node $i$ and internal node of the community. The value is negatively correlated with the edge-position reliability of the node. The tighter the connection between the internal node and the higher importance of edge-position of the node, the lower the reliability of the node.

\section{Link Reliability Analysis of Power Communication Net- work}

Links are an important part of power communication network. The reliable operation of the link will directly affect the reliability of the whole network. Links in power communication network are particularly prone to failures due to various human and natural factors, which affects the reliability of the power communication network. Traditional evaluation criteria for links include maximum flow, minimum path set, shortest path, minimum spanning tree, number of spanning tree, etc. These traditional evaluation methods are suitable for smallscale power communication network. When the network scale is increased, the calculation complexity of these indexes is greatly increased, which can not meet the timely evaluation of power communication network link reliability. In this section, a link reliability evaluation method of power communication network based on community structure is proposed, which combines the community structure model of power communication network

As shown in Fig. 8. In the absence of community division, all links in the network have no essential difference. However, after community partition, the nature of links $e_{1}$ and link $e_{2}$ has changed fundamentally. As a cross-community link, $e_{1}$ undertakes information exchange between communities. It

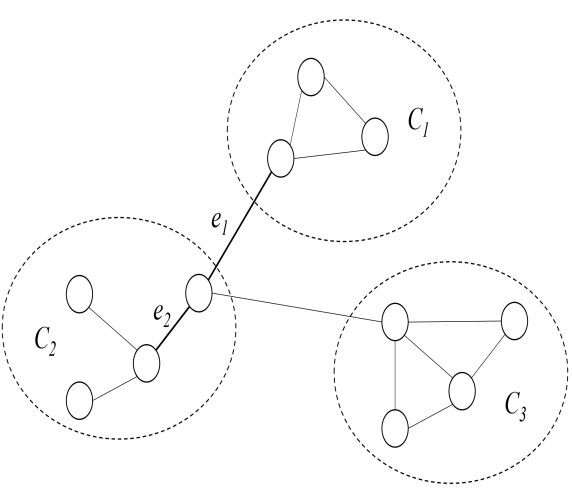

Fig. 8. Link comparison in community structure

plays an important role in information dissemination across different domains. If it is attacked, it will cause great loss to the integrity and connectivity of the whole network. The link $e_{2}$ is only the information transmission channel of the nodes within the community. No matter in the ability of information transmission or the importance, it is not as high as link $e_{1}$. Therefore, it is necessary to distinguish the reliability of links $e_{1}$ and $e_{2}$ when analyzing the reliability of links.

Through the above analysis, we proposes a link reliability evaluation index based on community structure (CER), which divides power communication network links into intercommunity links and intra-Community links. The reliability of different links is related to the reliability of their communities and their own reliability. The definition is as follows.

$\operatorname{CER}\left(e_{i j}\right)=\left\{\begin{array}{c}\sum_{k=1}^{|C|} \delta(i, k) C R\left(C_{k}\right) \times \sum_{k=1}^{|C|} \delta(j, k) C R\left(C_{k}\right) \\ \times \operatorname{ESR}\left(e_{i j}\right) C_{v_{i}} \neq C_{v_{j}} \\ \sum_{k=1}^{|C|} \delta(i, k) C R\left(C_{k}\right) \times \operatorname{ESR}\left(e_{i j}\right) \\ C_{v_{i}}=C_{v_{j}}\end{array}\right.$

$$
\begin{gathered}
\operatorname{ESR}\left(e_{i j}\right)=\frac{1}{B\left(e_{i j}\right)+1} \\
\delta(i, k)=\left\{\begin{array}{c}
1 i \in k \\
0 \text { otherwise }
\end{array}\right.
\end{gathered}
$$

We use $\operatorname{ESR}\left(e_{i j}\right)$ to measure the reliability of the link itself. It is expressed by the reciprocal of link median $B\left(e_{i j}\right)$ of link $e_{i j}$. Link median represents the proportion of the proportion of the number of paths that all the shortest paths pass through a link to the total number of shortest paths. It is a very important global geometric quantity, which is usually used to measure the reliability and importance of links. When the two ends of a link are located in different community, the reliability of the link is determined by the community reliability of the two ends and the reliability of the link itself. When the two ends of the link are in the same community, the reliability of the link is only determined by the reliability of its community.

\section{EXPERIMENT}

In order to measure the design rationality of reliability index of nodes (links), different attack models are used to remove 
attack on different nodes (links) of power communication network. Then the rationality of reliability index is evaluated by comparing the performance degradation of the network under different attack modes.

\section{A. Network Performance Evaluation Index}

When a node (link) of the power communication network is removed, the network performance will decrease. The greater the network performance decreases, the lower the reliability of the node (link) is. In this section, the performance of power communication network is evaluated from two aspects of network connectivity efficiency and connectivity.

Efficiency function is a function to measure connectivity efficiency for complex networks. Which describes the average value of "communication efficiency" between any two different nodes in the network. When any nodes (links) in power communication network are destroyed, the efficiency of the whole network decreases accordingly. According to different proportion and different ways to attack the nodes in the network, we can get the whole network efficiency of the power communication network under attack, so as to evaluate the tolerance degree of different nodes (links) and the damage degree of different nodes (links) to the whole network efficiency under attack.

$$
\begin{aligned}
E(G) & =\frac{1}{N(N-1)} \sum_{i, j \in G, i \neq j} \mathcal{E}_{i j} \\
& =\frac{1}{N(N-1)} \sum_{i, j \in G, i \neq j} \frac{1}{d_{i j}}
\end{aligned}
$$

Where $N$ is the number of nodes in the network. $\varepsilon_{i j}$ represents the communication efficiency between the two nodes. It is inversely proportional to the shortest distance between two nodes. When $i$ and $j$ are not connected, $\varepsilon_{i j}=0 . E(G)$ is the average communication efficiency between any two nodes of the whole network. When a communication node or link is removed, the performance of the whole network will be reduced, therefore, the value of the efficiency function will be reduced.

For the power communication network, the connectivity of the whole network is also a key factor to evaluate the performance of the power communication network. Due to the removal of nodes or edges, the power communication network may be divided into multiple connected components from a connected network, which seriously affect the network performance. In order to evaluate the network connectivity, we introduce the maximum connectivity $S(G)$, which is defined as the ratio of the number of nodes in the maximum connectivity component of the network after the power communication network node (link) fails and the total number of nodes in the power communication network. The formula of the maximum connectivity $E(G)$ is

$$
S(G)=\frac{n_{r}}{N}
$$

Where $N$ is the number of nodes in the network and $n_{r}$ is the number of nodes with the maximum connectivity component.
In order to measure the connectivity efficiency and connectivity, the comprehensive network performance evaluation index $C N P$ is introduced, the index definition is as follows:

$$
\begin{aligned}
C N P(G) & =E(G) \times S(G) \\
R & =\frac{C N P(G)}{C N P_{0}(G)}
\end{aligned}
$$

The size of network comprehensive performance index often changes with the change of network scale. Therefor, for the same network, its initial value has no effect on the change of network efficiency. In order to reflect the changing trend of network performance after being attacked, we introduce the relative efficiency ( $R$ value) to evaluate the declining trend of network efficiency, where $C N P_{0}(G)$ represents the comprehensive performance value when the network is not attacked.

\section{B. Attack Model}

The attack model used in this paper includes two models: random attack and deliberate attack. The attack process is to select certain nodes and links to remove in turn, to calculate the decline of the entire network performance of the power communication network. Random attacks are used to simulate random failures of nodes and links in the power communication network. In algorithm simulation, the principle of random number will be used to fail the specified nodes and links to simulate the attack; a deliberate attack is a point-specific attack with a special nature in a power communication network. Compare the changes in network performance after attack. During the simulation process, two kinds of deliberate attacks will be carried out on the node link, namely, high mesa attack and low reliability attack. For example, for example, high mesotype node attack is at some point, the serial number of the node is sorted from large to small by the number size of the node. And two node collections are established, respectively, the unattacked node collection and the self-attacked node collection, when attacked, the node with the highest number of collection mediations in the collection of never-hit nodes is placed in the collection of the attacked nodes, and the edge associated with the node on the network topology is removed, and observe before and after the removal of the nodes. The degree of change of the $r$ value of network relative efficiency.

\section{Result analysis}

This simulation is based on the backbone network ARPA [39] of the famous network 1979's Arpanet. It is a mediumsized network with 20 nodes and 32 edges. The result of community mining for Red ARPA is shown in Fig. 9 by our proposed algorithm.

As shown in Fig.8, nodes $\{1,2,3,4,4,8,14,16\}$ belong to community 1 , nodes $\{7,13,15,20\}$ belong to community 2 , nodes $\{5,6,9,11,12,17\}$ belong to community 3 , and nodes $\{10,18,19\}$ belong to community 4 . The reliability of the community can be calculated according to formula (4) as shown in TABLE I. 


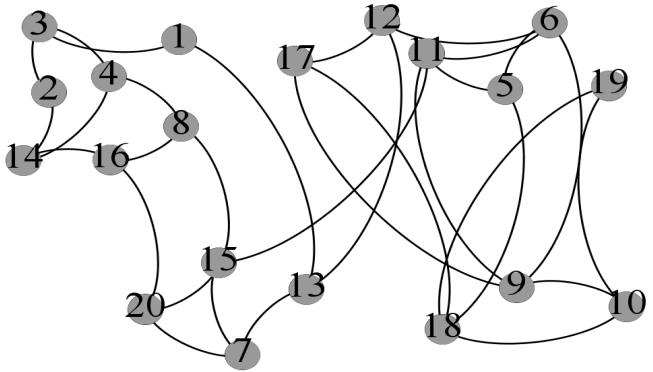

Fig. 9. Community detection Results of the network Red ARPA

TABLE I

RELIABILITY OF EACH COMMUNITY

\begin{tabular}{|c|c|c|c|c|}
\hline Community Number & 1 & 2 & 3 & 4 \\
\hline$C V\left(C_{i}\right)$ & 0.2 & 0.33 & 0.27 & 0.2 \\
\hline
\end{tabular}

TABLE II and TABLE III lists the reliability of each node and each edge.

The seven nodes with the lowest reliability are $\{5,12$, $13,15,17,18\}$. Most of these nodes are bridging nodes between associations, which are responsible for the exchange of information between the associations, and are critical nodes in the entire power communication network, which can cause great damage to the entire network. Causes a significant decrease in the performance of power communication network.

The seven links with the lowest reliability are $(9,10),(1,13)$, $(8,15),(17,18),(5,18),(12,13),(11,15)$. Most of these links are associative links between communities, and the reliability of these links to associations is low. The disconnection of such links will damage the connectivity of the whole power communication network, and the communities are easy to form isolated islands, resulting in the inaccessibility of information transmission. The protection of such links helps to ensure the connectivity of the whole network.

In order to verify the rationality of the reliability of the calculated nodes (links). Random, low-reliability and highbetweenness attacks on the nodes (links) with low reliability in the network. The changes of network performance under different attack modes are compared. The nodes and edges selected based on the three attack modes in the simulation process are shown in TABLE IV respectively.

According to the sequence of attack nodes (links) in the three attack modes selected above, the network performance decline curve of the number of attack nodes (links) is drawn to detect the impact of different nodes (links) on the overall network performance. The curve is shown in the following figure.

From Fig. 10, it can be seen that the relative efficiency $R$ of the network decreases faster than that of random attacks by deliberately attacking nodes with high-betweenness or low reliability. This shows that the power communication

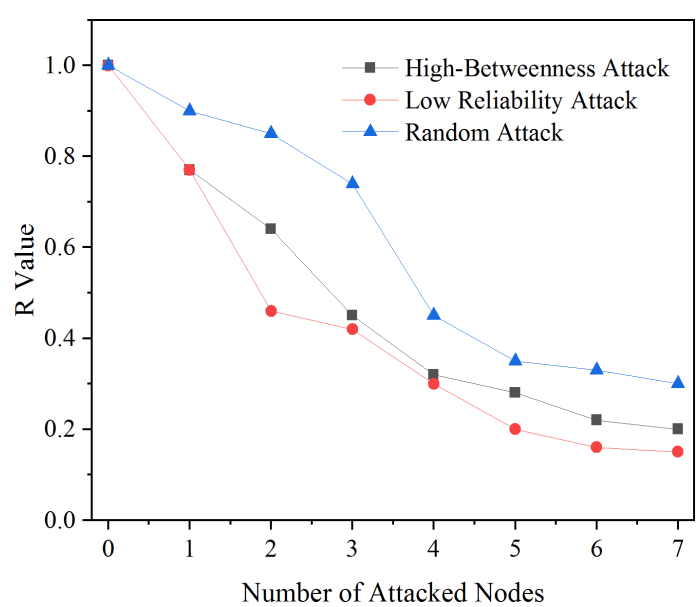

Fig. 10. $\mathrm{R}$ value under three attacks on nodes

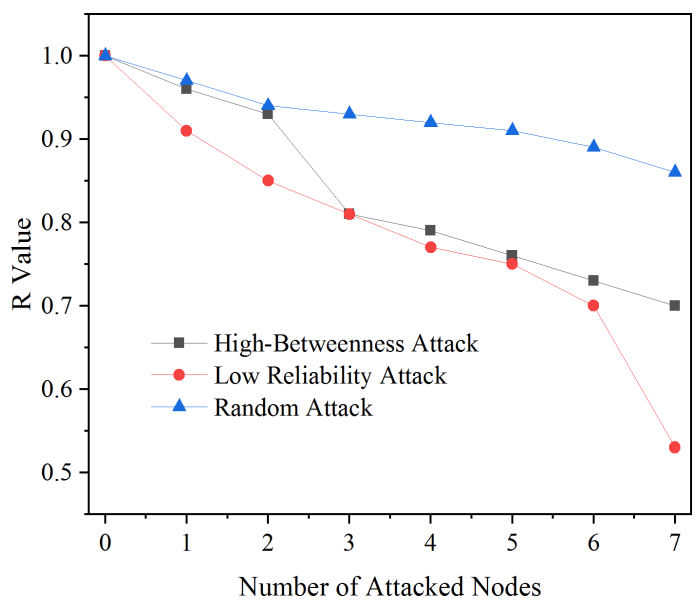

Fig. 11. R Value under three attacks on links

network is vulnerable to deliberate attacks, and accords with the robust and fragile scale-free characteristics of the power communication network. the power communication network has high median or can be attacked. When the reliability of nodes is low, the decline trend of $R$ value curve is more obvious. Attacking these nodes will have a huge impact on the network performance. Compared with attacking highbetweenness nodes, attacking low-reliability nodes will have a more obvious impact on the network performance, especially after attacking some low-reliability nodes, the connected network becomes many uncorrelated connected components. The performance of the network decreases obviously when the connected components are correlated. It can be concluded that the index presented in this paper can be used as the evaluation index of node reliability.

As shown in Fig.11, similar to node attack, this paper attacks links in the network in three ways, namely random attack, deliberate attack on edges with high link median and deliberate attack on low-reliability links evaluated by this index. From the above figure, we can see the impact of deliberate attack on network performance. Greater. In deliberate attacks, the damage of low-reliability links has a greater impact on network performance, and the decline of value curve is larger than 
TABLE II

RELIABILITY OF EACH NODE

\begin{tabular}{|c|c|c|c|c|c|c|c|}
\hline Community Number & $C N R$ & Community Number & $C N R$ & Community Number & $C N R$ & Community Number & $C N R$ \\
\hline 1 & 0.033 & 2 & 0.067 & 3 & 0.067 & 4 & 0.067 \\
\hline 5 & 0.027 & 6 & 0.068 & 7 & 0.110 & 8 & 0.033 \\
\hline 9 & 0.018 & 10 & 0.027 & 11 & 0.03 & 12 & 0.027 \\
\hline 13 & 0.017 & 14 & 0.067 & 15 & 0.009 & 16 & 0.033 \\
\hline 17 & 0.027 & 18 & 0.027 & 19 & 0.1 & 20 & 0.033 \\
\hline
\end{tabular}

TABLE III

RELIABILITY OF EACH LINK

\begin{tabular}{|c|c|c|c|c|c|c|c|}
\hline $\begin{array}{c}\text { Link } \\
\text { number }\end{array}$ & CER & $\begin{array}{c}\text { Link } \\
\text { number }\end{array}$ & CER & $\begin{array}{c}\text { Link } \\
\text { number }\end{array}$ & CER & $\begin{array}{c}\text { Link } \\
\text { number }\end{array}$ & $C E R$ \\
\hline$(1,2)$ & 0.194 & $(1,3)$ & 0.197 & $(2,14)$ & 0.190 & $(2,3)$ & 0.197 \\
\hline$(3,4)$ & 0.191 & $(4,14)$ & 0.193 & $(4,8)$ & 0.184 & $(5,18)$ & 0.051 \\
\hline$(5,6)$ & 0.266 & $(5,11)$ & 0.258 & $(6,11)$ & 0.257 & $(6,12)$ & 0.259 \\
\hline$(6,9)$ & 0.234 & $(7,13)$ & 0.320 & $(7,15)$ & 0.314 & $(7,20)$ & 0.315 \\
\hline$(8,16)$ & 0.199 & $(8,15)$ & 0.059 & $(9,10)$ & 0.050 & $(9,17)$ & 0.255 \\
\hline$(10,18)$ & 0.199 & $(10,19)$ & 0.191 & $(11,15)$ & 0.078 & $(12,13)$ & 0.084 \\
\hline$(12,17)$ & 0.236 & $(14,16)$ & 0.191 & $(15,20)$ & 0.303 & $(16,20)$ & 0.063 \\
\hline$(17,18)$ & 0.051 & $(18,19)$ & 0.191 & $(1,13)$ & 0.050 & $(9,11)$ & 0.254 \\
\hline
\end{tabular}

that of R-value curve under high-median attacks. Emphasis on protection of such low-reliability links in the network helps to ensure the stable operation of the network. Thus, the power communication network links set in this paper can be used. Reliability model has certain reference value for the protection of power communication network.

\section{The Coping Strategies in the Face of DELIBERATE ATTACKS}

Through the above simulations, it can be seen that when the key nodes or links in power communication network are deliberately attacked, the performance of power communication network will decrease rapidly. More seriously, if several key nodes or links are damaged at the same time, the whole power communication network will be paralyzed. Therefore, strengthening the protection of key nodes (links) plays a key role in improving the stability of communication network. In the real world, there are usually several measures to protect the key nodes and links.

- For power communication network nodes with low reliability, they can be protected by replacing equipment in time and increasing equipment redundancy (such as hot standby for communication sites, switching between master and backup, etc.). Although these methods do not improve the robustness of the network itself, they are greatly improved from the angle of attack-defense. It reduces the probability of attack on low reliability nodes.

- For links with low reliability, the strategy of increasing the number of edges can be used to reduce the critical degree of links in the whole network. By adding communication lines, the robustness of power communication network can be effectively improved and effectively avoid the sharp drop in network efficiency caused by deliberate attacks on low-reliability links.

- From the point of view of power communication network business, the business pressure on nodes (links) with lower reliability should be reduced. The business importance rating of the business characteristics of the power communication network is graded, and for key business, if the business is transmitted through a link (node) with lower reliability, it needs to be re-routed to avoid these low reliability nodes and links. In this way, it can not only ensure the normal operation of key business, but also reduce the business pressure of these nodes and links.

\section{CONCLUSION}

In this paper, firstly, the topological structure of power communication network is analyzed, and the obvious community structure of power communication network is confirmed. Then, the improved parallel community mining algorithm is used to mine the community structure of power communication network. Based on the community structure, the reliability evaluation indicator of node (link) of power communication network is constructed. Compared with the traditional reliability indicator, our indicator not only consider the influence of the environment of nodes (links) on the structure of power communication network, but also improve the reliability evaluation rate of nodes (links), it can be applied to the reliability evaluation of power communication network nodes (links).

In order to verify the rationality of the reliability indicator of the node (link), this paper uses three methods of random attack, low reliability and high betweeness to attack the designated node (link) of the power communication network, and compares the network efficiency change after the attack. In the simulation process, it is found that under the deliberate attack strategy of low reliability nodes (links), the network performance of the communication network decreases faster. The damage of these nodes (links) has more obvious effect on the performance of the network, and the rationality of the reliability evaluation indicator of this paper is verified.

\section{REFERENCES}

[1] X. Fang, S. Misra, G. Xue, and D. Yang, "Smart grid - the new and improved power grid: A survey," IEEE Communications Surveys and Tutorials, vol. 14, no. 4, pp. 944-980, 2012.

[2] V. C. Gungor, D. Sahin, T. Kocak, S. Ergut, C. Buccella, C. Cecati, and G. P. Hancke, "Smart grid technologies: Communication technologies and standards," IEEE Transactions on Industrial Informatics, vol. 7, no. 4, pp. 529-539, 2011.

[3] P. Yao, G. Liu, and Y. Liu, "Smart meter based on smart energy profile for zigbee and power line communication connectivity-enabled home area network," Applied Mechanics and Materials, vol. 599-601, p. 4, 2014.

[4] O. Vukovic, K. C. Sou, G. Dan, and H. Sandberg, "Network-aware mitigation of data integrity attacks on power system state estimation," IEEE Journal on Selected Areas in Communications, vol. 30, no. 6, pp. 1108-1118, 2012. 
TABLE IV

ATTACK NODE AND LINK INSTANCE

\begin{tabular}{|c|c|c|}
\hline Attack mode & Selecting nodes & Selecting link number \\
\hline High median attack & $\{1,7,11,12,13,15,17\}$ & $\{(8,15),(1,13),(9,10),(12,13),(17,18),(11,15),(12,17)\}$ \\
\hline Low reliability attack & $\{5,9,12,13,15,17,18\}$ & $\{(9,10),(1,13),(8,15),(17,18),(5,18),(12,13),(11,15)\}$ \\
\hline Random attack & $\{3,5,6,7,12,17,19\}$ & $\{(6,9),(7,15),(3,4),(15,20),(10,18),(1,13),(7,20)\}$ \\
\hline
\end{tabular}

[5] M. Eliasson, "The challenge in building a global ground communication network for satellite and space operations using the international telecommunication industry." in Spaceops, 2013.

[6] M. Lei, X. Zhang, B. Yu, and X. Dong, "Power and discrete rate adaptation in ber constrained wireless powered communication networks," IET Communications, vol. 12, no. 18, pp. 2213-2221, 2018.

[7] D. Liu, J. I. Xingpei, G. Chen, B. Wang, and D. Wei, "Link addition strategy based on complex network theory for power communication network," Electric Power Automation Equipment, 2016.

[8] Rahman, F. Abdul, Varuttamaseni, Athi, Lee, C. John, Kintner-Meyer, and Michael, "Application of fault tree analysis for customer reliability assessment; of a distribution power system," Reliability Engineering \& System Safety, vol. 111, no. 111, pp. 76-85, 2013.

[9] P. L. Joskow and J. Tirole, "Transmission rights and market power on electric power networks," Cepr Discussion Papers, vol. 31, no. 3, pp. 450-487, 2000

[10] Y. K. Lin and C. T. Yeh, "Maximal network reliability for a stochastic power transmission network," Reliability Engineering \& System Safety, vol. 96, no. 10, pp. 1332-1339, 2017.

[11] T. Lu, F. Han, Z. Zhao, and C. Xiang, "Analysis of the electric field and ion current density under ultra high-voltage direct-current transmission lines based on finite element method," IEEE Transactions on Magnetics, vol. 43, no. 4, pp. 1221-1224, 2007.

[12] H. Lin, S. Sambamoorthy, S. Shukla, J. Thorp, and L. Mili, "Power system and communication network co-simulation for smart grid applications," in Innovative Smart Grid Technologies, 2011.

[13] C. Ziogou, D. Ipsakis, C. Elmasides, F. Stergiopoulos, S. Papadopoulou, P. Seferlis, and S. Voutetakis, "Automation infrastructure and operation control strategy in a stand-alone power system based on renewable energy sources," Journal of Power Sources, vol. 196, no. 22, pp. 94889499, 2011.

[14] C. Pizzuti, "Evolutionary computation for community detection in networks: A review," IEEE Transactions on Evolutionary Computation, vol. 22, no. 3, pp. 464-483, 2018.

[15] Q. Li, J. Zhong, Q. Li, C. Wang, and Z. Cao, "A community merger of optimization algorithm to extract overlapping communities in networks," IEEE Access.

[16] M. E. Newman and M. Girvan, "Finding and evaluating community structure in networks." Physical Review E Statistical Nonlinear and Soft Matter Physics, vol. 69, no. 2, p. 026113, 2004.

[17] C. Aaron, M. E. J. Newman, and M. Cristopher, "Finding community structure in very large networks," Physical Review E, vol. 70, no. 2, p. 066111, 2004.

[18] X. Liu, Z. Xie, and D. Y. Yi, "Community detection by neighborhood similarity," Chinese Physics Letters, vol. 29, no. 4, pp. 48 902-48 905(4), 2012.

[19] J. Li, X. Wang, and Y. Cui, "Uncovering the overlapping community structure of complex networks by maximal cliques," Physica A Statistical Mechanics and Its Applications, vol. 415, pp. 398-406, 2014.

[20] S. Gregory, "A fast algorithm to find overlapping communities in networks," 2008.

[21] X. Tong, Y. Zhang, and Y. Dai, "From public communication network to public computing communication network," Telecommunications Science, 2010.

[22] D. Reichelt and P. Linser, "A study of an iterated local search on the reliable communication networks design problem," in Evoworkkshops. Evobio, Evocomnet, Evohot, Evoiasp, Evomusart, and Evostoc, 2005.

[23] D. Gambhir, I. T. Frisch, and M. Post, "Automated communication network software fault isolation," in IEEE International Conference on Systems, 1991

[24] S. H. Sun, Z. D. Zhao, and L. H. Yang, "Disjoint network reliability index algorithm in the application of the power communication network," Advanced Materials Research, vol. 684, pp. 575-578, 2013.

[25] S. H. Lee, H. E. Kim, K. S. Son, S. M. Shin, S. J. Lee, and H. G. Kang, "Reliability modeling of safety-critical network communication in a digitalized nuclear power plant," Reliability Engineering and System Safety, vol. 144, pp. 285-295, 2015.
[26] Z. D. Zhao, Y. Y. Lou, Y. D. Zhang, and Y. J. Wang, "Structure of reliability evaluation model for electric power communication network," Electric Power Technology, 2010

[27] L. Jie, L. Gang, and M. Xin, "Analysis of power grid control service information and communication network reliability model," in Second International Conference on Modeling, 2010.

[28] A. S. Dulesov, D. Y. Karandeev, and N. V. Dulesova, "Reliability analysis of distribution network of mining enterprises electrical power supply based on measure of information uncertainty," in Iop Conference Series: Earth and Environmental Science, 2017.

[29] Z. D. Zhao, Y. Y. Lou, J. H. Ni, and J. Zhang, "Rbf-svm and its application on reliability evaluation of electric power system communication network," in International Conference on Machine Learning and Cybernetics, 2009.

[30] D. H. O. Mcqueen, P. R. Hyland, and S. J. Watson, "Monte carlo simulation of residential electricity demand for forecasting maximum demand on distribution networks," IEEE Transactions on Power Systems, vol. 19, no. 3, pp. 1685-1689, 2004.

[31] J. H. Yang, X. D. Peng, and Y. J. Chao, "Reliability prediction of power communication network based on bp neural network optimized by genetic algorithm," 2017.

[32] K. Zhang, W. Shi, H. Zhu, and T. Başar, "Distributed equilibriumlearning for power network voltage control with a locally connected communication network," 2018.

[33] X. Wei and H. Wang, "Application study on power communication network for intelligent community," Microcontrollers and Embedded Systems, 2013.

[34] A. D. Mccarthy, T. Chen, R. Rudinger, and D. W. Matula, "Metrics matter in community detection," 2019.

[35] H. M. Cheng, Y. Z. Ning, Z. Yin, C. Yan, X. Liu, and Z. Y. Zhang, "Community detection in complex networks using link prediction," Modern Physics Letters B, vol. 32, no. 3, p. 1850004, 2018.

[36] M. Gong, L. Ma, Q. Zhang, and L. Jiao, "Community detection in networks by using multiobjective evolutionary algorithm with decomposition," Physica A Statistical Mechanics and Its Applications, vol. 391, no. 15 , pp. $4050-4060,2012$.

[37] I. Lobov and S. Ivanov, "Unsupervised community detection with modularity-based attention model," 2019.

[38] V. D. Blondel, J. L. Guillaume, R. Lambiotte, and E. Lefebvre, "Fast unfolding of communities in large networks," Journal of Statistical Mechanics, vol. 2008, no. 10, pp. 155-168, 2008.

[39] S. Cohn, Arpanet routing, 1990. 